\title{
Dynamic Load Equilibration for Cyclic Applications in Distributed Systems
}

\author{
Siegfried Höfinger \\ Department of Theoretical Chemistry and Molecular Structural Biology, \\ Molecular Dynamics and Biomolecular Simulation Group, \\ University of Vienna, \\ Währingerstr. 17, \\ A-1090, Vienna, Austria \\ sh@mdy.univie.ac.at \\ http://www.mdy.univie.ac.at
}

\begin{abstract}
A Wide Area Network cluster consisting of 5 UNIX-type machines of different architectures and different operating systems is set up via PVM 3.4.3. The 5 nodes are distributed over 4 European research centres located in France, Italy and Austria and only secure shell-type internode communication is allowed. The recently developed concept of Speed Weighted Load Balancing is employed when using GREMLIN a Quantum Chmistry program - for the electronic structure calculation of alanine. In contrast to the previous static concept, a new dynamic functionality is added, that allows for runtime re-equilibration of all the participating hosts. Even complicated events, such as crash down of contributing hosts may be adressed from this new, dynamic, version of Speed Weighted Load Balancing, without the loss of intermediary data, or a considerable deviation from optimal host performance.
\end{abstract}

\section{Introduction}

Distributed Computing has become a major focus in current research and development efforts [1] [2], and it is especially those applications, that efficiently can make use of the steadily growing number of accessible internet computers - nowadays simplified termed "the grid" - that are said to deserve particular attention and need all the concentrated development initiative that is possible. Supercomputer applications in science and technology seem to become feasible in an unforeseen dimension and thus insight into fundamental principles of nature might be gained, if - and only if - these applications really succeed in taking full advantage of all the computational power inherent in the grid of today. Without any doubt Computational Chemistry in general, and Quantum Chemistry in particular, may be considered to be one such example for a scientific discipline, that needs as much computational power as possible, in order to be able to answer the more and more complex growing queries emerging from bio-sciences, chemical industry, etc. Exactly with this future needs in mind GREMLIN [3], a Quantum Chemistry program, that solves the time independent Schrödinger 
equation [4] according to the Hartree Fock Method [5] [6], has been developed with special emphasis on parallel features for shared memory multiprocessor machines [7] as well as distributed cluster architectures of either homogenous type [3] or even of heterogenous character [8].

In a previous article it was shown [8] that after taking into account the different node performances of all the individual hosts that formed up a $W A N$ cluster (Wide Area Network cluster), at the very basic stage of recursive ERIcomputation (Electron Repulsion Integrals), which constitutes the major computational task in any Quantum Chemistry program, nearly optimal performance and speed-up could have been established. This previous concept of Speed Weighted Load Balancing $(S W L B)$ could partition the net amount of $E R I$ work into appropriate node-specific fractions based on an input-provided list of relative speed factors, which simply are the proportional constants of all the hosts performances with respect to the slowest machine. However, this process was envoked only once in the beginning of the whole iterative procedure and thus any later change in either network or host conditions would not have been recognized, nor could have been counterbalanced accordingly. Therefore, in this present work additional dynamic capability of $S W L B$ shall be described, which now offers fast and powerful reactivity to any kind of change in the $W A N$ - cluster environment, where the measures of re-equilibrating become active in between two subsequent iteration steps of the calculation. It is a frequently observed characteristic in many large-scale scientific applications, that they repeat a certain major step several times until either an accuracy criterion is met, or an initially defined overall number of cycles are completed. Therefore it would be of great advantage, if in between these repeated cycles possible changes in cluster conditions could be recognized and handled accordingly.

In the remainder of this article a presentation of an extension to static $S W L B$ shall be given, that actually will result in enabling all mentioned dynamic functionality and therefore should contribute considerably to an improvement of efficiency in distributed computing solutions.

\subsection{Problem Description}

In the following a brief summary is given that should outline the principal problem arising with electronic structure calculations and their intrinsic need for computational power. The key process - and also the main time-consuming one - is the evaluation of ERIs, the Electron Repulsion Integrals, which are 6-dimensional, 4-center integrals over the basis functions $\varphi_{i}$

$$
E R I=\iint_{\boldsymbol{r}_{\mathbf{1}}} \varphi_{\boldsymbol{r}_{\mathbf{2}}}\left(\boldsymbol{r}_{\mathbf{1}}\right) \varphi_{j}\left(\boldsymbol{r}_{\mathbf{1}}\right) \frac{1}{\left|\boldsymbol{r}_{\mathbf{2}}-\boldsymbol{r}_{\mathbf{1}}\right|} \varphi_{k}\left(\boldsymbol{r}_{\mathbf{2}}\right) \varphi_{l}\left(\boldsymbol{r}_{\mathbf{2}}\right) \boldsymbol{d} \boldsymbol{r}_{\mathbf{1}} \boldsymbol{d} \boldsymbol{r}_{\mathbf{2}}
$$

. The basis functions $\varphi_{i}$ are expanded in a series over Primitive Gaussians $\chi_{j}$

$$
\varphi_{i}(\boldsymbol{r})=\sum_{j} d_{i, j} \chi_{j}(\boldsymbol{r})
$$


which typically are Cartesian Gaussian Functions [10] [11] located at some position $\left(A_{x}, A_{y}, A_{z}\right)$ in space.

$$
\chi_{j}(\boldsymbol{r})=N_{j}\left(x-A_{x}\right)^{l}\left(y-A_{y}\right)^{m}\left(z-A_{z}\right)^{n} e^{-\alpha_{j}(\boldsymbol{r}-\boldsymbol{A})^{2}}
$$

An S-type basis function will consist of primitive gaussians with $l=m=n=0$, a P-type however of primitives with $l+m+n=1$, which may be solved at 3 different ways, either $l=1$ and $m=n=0$, or $m=1$ and $l=n=0$, or $n=1$ and $l=m=0$. A D-type specification will likewise mean $l+m+n=2$ and similarly F-type $l+m+n=3$.

The principal number of ERIs to be considered grows with the 4th power of the number of basis functions, and the latter is proportional to the number of atoms in the molecule. Because modern Quantum Chemistry made clear, how important it is to use extensive, high-quality basis sets, the according number of regarded ERIs very soon exceeds conventional RAM and diskspace limits and thus becomes the only limiting factor at all. For example, a simple, small molecule like the amino acid alanine (13 atoms), that has been used in this study, at a basis set description of aug-cc-pVDZ quality [12] [13] (213 basis functions of S, P and D type, 4004 primitive gaussians) leads to a theoretical number of approximately $260 \times 10^{6} \mathrm{ERI}$, which requires about 2.1 GigaByte of permanent or temporary memory and goes far beyond conventional computational resources.

However, an approach called "direct methodology" provides a way to still deal with much larger problem sizes by exploiting the fact that there is partial independence in the mathematical action of all these ERIs. In detail this means, that first a certain logical block of related ERIs is calculated recursively, where all complicated ERI-types $(l+m+n>0)$ may be deduced from the simpler $\left(S_{i}, S_{j} \mid S_{k}, S_{l}\right)$ type. In the next step the action of the entire block on all the corresponding Fock-matrix elements — from which there are only a number of (number of basisfunctions $)^{2}$ - is considered, and then the new block of $E R I s$, which overwrites the old one, is formed. Therefore only a small amount of working memory is permanently involved.

Nevertheless one has to respect a hierarchic structure in spawning the space to the final primitive cartesian gaussian functions $\chi_{j}$, where, following the notation introduced in [3], a certain center $\mathrm{i}$ refers to an according block of contracted shells $\rightarrow(\mathrm{j})$...(k), from which each of them maps onto corresponding intervals of basis functions $1 . . . \mathrm{m}$ and the latter are expanded from primitive cartesian gaussian functions $\chi_{j}$ as seen from equation (2). Therefore, a particular centre quartette i1 i2 i3 i4 will entirely define a complete block of integrals, which must be solved together at once. This also becomes the major problem when partitioning the global amount of integrals into equally sized portions, because these blocks may be of considerable different size and one needs to find a well-balanced distribution of centre-quartettes over nodes, that ideally will cause all the various participating hosts to work equally long for the completion of their assigned blocks of integrals. 


\subsection{Speed Weighted Load Balancing, Static - Dynamic}

The goal here is to distribute fractions of the global amount of ERIs over all the participating nodes forming up the $W A N$-cluster. If this happens to be done in a way, that all the hosts will actually require exactly the same time to complete their partial tasks, the calculation will be accelerated optimally and one could not do better. Unfortunately a certain block of ERIs cannot be considered an independent unit, but is a hierarchic subsection of a set of contracted shells (j)...(k) and these again are a subspace of atomic centres i1 i2 i3 i4 and only the last property will be sent forward to the nodes, which will start their work based on received centre-combinations independently and autonomously.

One cannot simply divide the overall numer of theoretical centre quartettes into as many equal sized fractions as there are hosts involved and expect optimal speed up [3]. On the contrary one will have to weigh a certain combination of atomic centres, according to the hierarchic ERI-work it will induce. This weightening can be done by estimating the total number of deducable ERIs for a certain centre quartette and this is usually done within a neglectable amount of CPU-time. In case of different node performance, which is the general case for $W A N$ clusters, the weightening must only be enhanced by the additional factor of per-node-speed, where the relative speed of each node is a multiple of the slowest host speed, but principally the same scheme may be employed [8].

Static Speed Weighted Load Balancing therefore was the implementation of the following steps:

1. Determine the number of participating hosts (NMB) and their according relative speed factors (SPEED[I]). The speed factor is the relative performance of a particular node related to the slowest CPU. So it will either become 1.0 (weakest node), or greater than 1.0 for faster CPUs.

2. Estimate the net amount of computational work (GLOBAL WORK) at the level of quartettes of primitive gaussians to be considered.

3. Form a unit portion (PORTN) of the dimension of

$$
\text { PORTN }=\frac{\text { GLOBAL WORK }}{\sum_{\mathrm{I}=1}^{\mathrm{NMB}} \operatorname{SPEED}[\mathrm{I}]}
$$

4. Loop again over all quartettes of centres and the related contracted shells and basis functions and primitive gaussians either, as if you were calculating GLOBAL WORK, and successively fill the upcoming pair lists for centre quartettes until in the work estimation variable (usually a simple counter, incremented for each new quartette of primitive gaussians) becomes of the size of PORTN*SPEED[I]; then leave the current pair list writing for node I and switch forward to the next node and start with setting up pair lists for this one.

In the Dynamic variant of $S W L B$ essentially the same steps shall be performed, however, now the whole route of instructions shall be repeated in each of the iterative cycles of the calculation. 
Suppose there was a second, completely unrelated process started at some of the nodes, e.g. one, that has got a single CPU only. Then the according SPEED[I] would become just half of SPEED[I], because the rest of the CPU-time would be consumed by the second, unrelated process, that has nothing to do with the cluster calculation. As a consequence, when it comes to summing up all the node data for an intermediary iteration result, all the other nodes $\mathrm{J} \neq \mathrm{I}$ would have to wait for the delayed arrival of the results of node I, and this would go on likewise until the end of the calculation due to the static distribution of the workload.

Only from repeated calls to the partitioning module in each of the iteration cycles, such a serious change in host conditions could be recognized and controlled, and this is what is done in Dynamic $S W L B$. The actual interference with the partitioning module is realized from modifying the speed factors SPEED $[\mathrm{I}]$, which means for the example above, that the value of SPEED $[\mathrm{I}]$ will be corrected to $0.5^{*} \mathrm{SPEED}[\mathrm{I}]$ as long as the 2 nd process is active. The changed partitioning scheme would immediately lead to close-to-optimal cluster performance again.

In addition, even drastic changes in the cluster set-up may be covered from Dynamic $S W L B$. For example in the case of crash-down of a certain host I, one only needs to set its corresponding speed factor to $\operatorname{SPEED}[\mathrm{I}]=0$, and the calculation will continue with reassigning the current iteration data, but without having to restart the run.

\section{WAN Cluster Description}

The $W A N$-cluster was set up of five machines located at GUP LINZ (A) (2 nodes), $R I S T^{++}$SALZBURG (A) (1 node), ICPS STRASBOURG (F) (1 node) and G. Ciamician BOLOGNA (I) (1 node). Internode communication was realized from PVM (Parallel Virtual Machine, rel.3.4.3) [14] based on 1024 bit RSA authenticated ssh-connections. Individual benchmark runs on the small test system glycine/631g (10 centre, 55 basis functions) revealed initial $W A N$-cluster conditions appropriate for Static $S W L B$ as shown in table 1, where the network bandwith was measured between the individual nodes and the future master machine (node III). Speed factors were derived from the glycine-benchmark and reflect performance ratios with respect to the slowest machine (node I).

\section{Discussion}

Dynamic $S W L B$ was used for the $W A N$-cluster calculation of alanine/aug-ccpVDZ (13 atoms, 213 basis functions) and according speed factors were updated every second iteration. Static $S W L B$ (initial two iterations) would lead to a run time behaviour as shown in Fig. 1, where it must be noted that node III not only acts as the master machine, that receives all the data from the remaining hosts and does the serial part of the calculation, but also contributes his CPU to the parallel region. Arrival of parallel thread of node III in Fig. 1 as the first and fastest one is just accidentally. A similar representation on the same scale for Dynamic $S W L B$ resulted in the plots shown in Fig. 2 and Fig. 3. Comparison of 
Table 1. Speed-Factor and Network-Latency table for the $W A N$-cluster. Speed-Factors represent the relative performance of all the individual hosts in the $W A N$-cluster with respect to the slowest performing CPU (glycine/631g/-271.1538 Hartree in 17 iterations). Network bandwidth was obtained from measuring transfer rates between nodes and the future master-machine (node III).

\begin{tabular}{|c|c|c|c|c|c|}
\hline $\begin{array}{l}\text { Physical } \\
\text { Location }\end{array}$ & $\begin{array}{l}\text { Architecture/ } \\
\text { Clock Speed/ } \\
\text { RAM/2L-Cache }\end{array}$ & $\begin{array}{l}\text { Operating } \\
\text { System }\end{array}$ & $\begin{array}{l}\text { Relative } \\
\text { Speed } \\
\text { Factor }\end{array}$ & $\begin{array}{c}\text { Network } \\
\text { Bandwidth } \\
{[\mathrm{kB} / \mathrm{s}]}\end{array}$ & $\begin{array}{l}\text { Exp.Total } \\
\text { Comm. } \\
\text { Time }[\mathrm{s}]\end{array}$ \\
\hline $\begin{array}{l}\text { node I } \\
\text { G.C. BOLOGNA } \\
\text { Italy }\end{array}$ & $\begin{array}{l}\text { INTEL Dual PPro } \\
200 \mathrm{MHz} \\
256 \mathrm{MB} / 512 \mathrm{~KB}\end{array}$ & $\begin{array}{l}\text { LINUX } \\
2.2 .14\end{array}$ & 1.000 & 166 & 128 \\
\hline $\begin{array}{l}\text { node II } \\
\text { ICPS STRASBOURG } \\
\text { France }\end{array}$ & $\begin{array}{l}\text { MIPS R10000 } \\
200 \mathrm{MHz} \\
20 \mathrm{~GB} / 4 \mathrm{MB}\end{array}$ & $\begin{array}{l}\text { IRIX } 64 \\
6.5\end{array}$ & 1.767 & 608 & 35 \\
\hline $\begin{array}{l}\text { node III } \\
\text { GUP LINZ } \\
\text { Austria }\end{array}$ & $\begin{array}{l}\text { INTEL PII } \\
350 \mathrm{MHz} \\
128 \mathrm{MB} / 512 \mathrm{~KB}\end{array}$ & $\begin{array}{l}\text { LINUX } \\
2.2 .13\end{array}$ & 1.943 & - & - \\
\hline $\begin{array}{l}\text { node IV } \\
\text { GUP LINZ } \\
\text { Austria }\end{array}$ & $\begin{array}{l}\text { MIPS R12000 } \\
400 \mathrm{MHz} \\
64 \mathrm{~GB} / 8 \mathrm{MB}\end{array}$ & $\begin{array}{l}\text { IRIX } 64 \\
6.5\end{array}$ & 3.609 & 918 & 23 \\
\hline $\begin{array}{l}\text { node V } \\
\text { RIST SALZBURG } \\
\text { Austria }\end{array}$ & $\begin{array}{l}\text { ALPHA EV6 } 21264 \\
500 \mathrm{MHz} \\
512 \mathrm{MB} / 4 \mathrm{MB}\end{array}$ & $\begin{array}{l}\text { OSF I } \\
\text { V } 5.0\end{array}$ & 6.357 & 592 & 36 \\
\hline
\end{tabular}




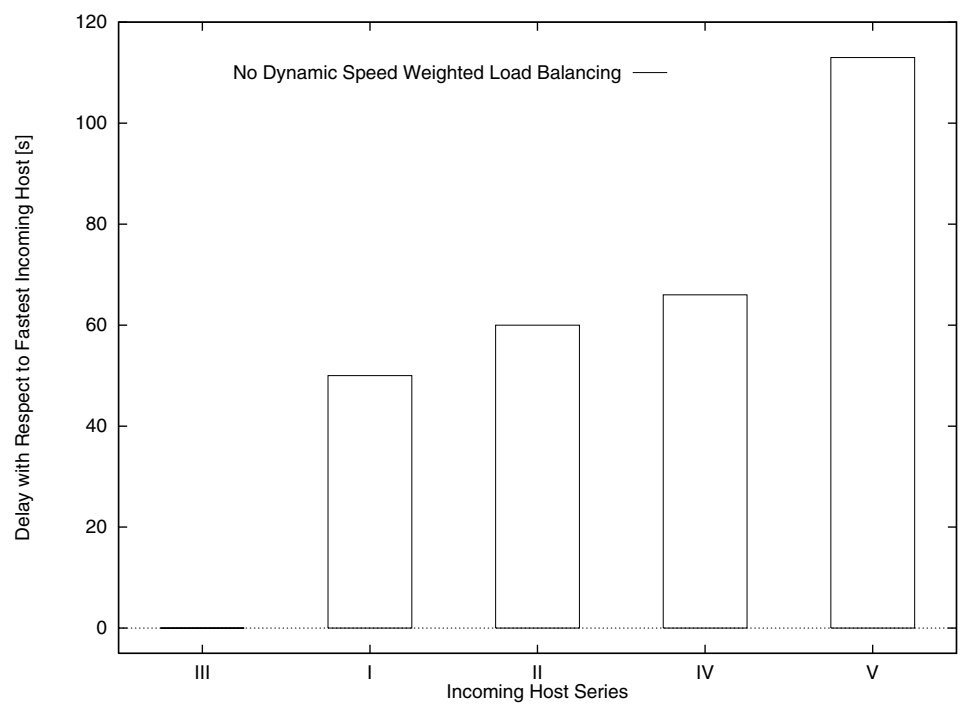

Fig. 1. Representation of the run time behaviour in Static SWLB for the iterative data collection phase of the Hartree Fock calculation of alanine/aug-cc-pVDZ with GREMLIN in a ssh-connected WAN-cluster, made of 5 far-distant machines.

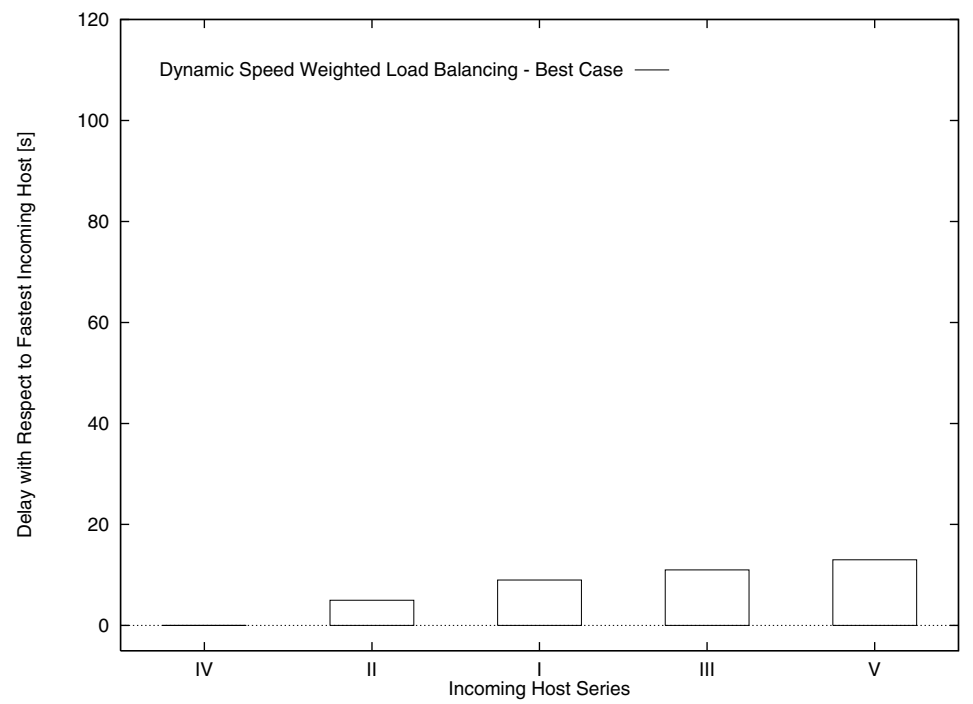

Fig. 2. Representation of the optimal run time behaviour in Dynamic $S W L B$ for the iterative data collection phase of the Hartree Fock calculation of alanine/aug-cc-pVDZ with GREMLIN in a ssh-connected $W A N$-cluster, made of 5 far-distant machines. 


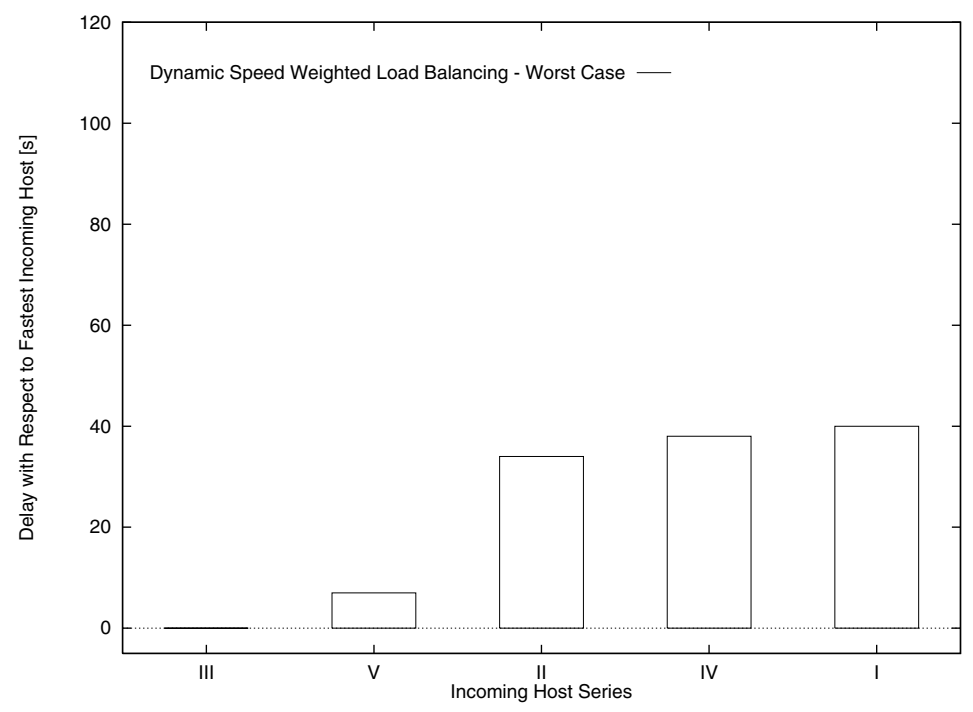

Fig. 3. Representation of the worst case run time behaviour in Dynamic $S W L B$ for the iterative data collection phase of the Hartree Fock calculation of alanine/aug-cc-pVDZ with GREMLIN in a ssh-connected $W A N$-cluster, made of 5 far-distant machines.

figures Fig.1 through Fig.3 shows a significant reduction of idle-time during the phase when all the nodes send their partial results of one particular iteration to the master node. From this a significant improvement of parallel performance may be concluded. Furthermore, it must be noted, that from the all in all 19 iterations needed for completion of the calculation (result: -321.7924 Hartree) 13 were best-case-like and only 6 were of worst-case character. As seen from figures Fig.1 through Fig. 3 the dynamic character of the workload equilibration also changes the order in which the individual nodes arrive at the master. Further event handling was simulated in a sperate run by suddenly setting speed factors of node II or III to zero and thus simulating a temporary breakdown of cluster nodes and in either case the calculation was continued successfully and the change in cluster environment was completely outbalanced within the first subsequent iteration.

\section{Conclusion}

Taking into account variable node performance in heterogenous $W A N$ cluster environments e.g. by the use of Dynamic Speed Weighted Load Balancing will substantially enhance overall performance. It also improves the stability and robustness of large grid-applications especially those that perform cyclic iterations and spend almost no time on communication. 


\section{Acknowledgement}

The author would like to thank Prof. Zinterhof from RIST ${ }^{++}$Salzburg, Prof. Volkert from GUP Linz Dr. Romaric David from ICPS Strasbourg and Prof. Zerbetto from Ciamician Bologna for providing access to their supercomputer facilities.

\section{References}

1. Czajkowski, K., Fitzgerald, S., Foster, I., Kesselman, C.: Grid Information Services for Distributed Resource Sharing. Proc. 10. IEEE Int. Symp. on High-Performance Distributed Computing IEEE Press (2001)

2. Allen, G., Foster, I., Karonis, N., Ripeanu, M., Seidel, E., Toonen, B.: Supporting Efficient Execution in Heterogeneous Distributed Computing Environments with Cactus and Globus. Proc. SC. 2001 (2001)

3. Höfinger, S., Steinhauser, O., Zinterhof, P.: Performance Analysis and Derived Parallelization Strategy for a SCF Program at the Hartree Fock Level. Lect. Nt. Comp. Sc. 1557 (1999) 163-172

4. Schrödinger, E.: Quantisierung als Eigenwertproblem. Ann. d. Phys. 79, 80, 81 (1926)

5. Hartree, D.R.: Proc. Camb. Phil. Soc., 24 (1928) 89

6. Fock, V.: Näherungsmethoden zur Lösung des Quantenmechanischen Mehrkörperproblems. Z. Phys. 61 (1930) 12662 (1930) 795

7. Höfinger, S., Steinhauser, O., Zinterhof, P.: Performance Analysis, PVM and MPI Implementation of a DSCF Hartree Fock Program. J. Comp. Inf. Techn. 8 (1) (2000) 19-30

8. Höfinger, S.: Balancing for the Electronic Structure Program GREMLIN in a Very Heterogenous SSH-Connected WAN-Cluster of UNIX-Type Hosts. Lect. Nt. Comp. Sc. 2074 (2001) 801-810

9. Obara, S., Saika, A.: Efficient recursive computation of molecular integrals over Cartesian Gaussian functions. J. Chem. Phys. 84 (7) (1986) 3963-3974

10. Davidson, E.R., Feller, D.: Basis Set Selection for Molecular Calculations. Chem. Rev., 86 (1986) 681-696

11. Shavitt, I.: The Gaussian Function in Calculations of Statistical Mechanics and Quantum Mechanics. Methods in Comp. Phys. ac. New York, 2 (1963) 1-44

12. Dunning Jr., T. H.: J. Chem. Phys. 90 (1989) 1007-1023

13. Woon, D. E., Dunning Jr., T. H.: J. Chem. Phys. 98 (1993) 1358-1371

14. Geist, G., Kohl, J., Manchel, R., Papadopoulos, P.: New Features of PVM 3.4 and Beyond. Hermes Publishing, Paris Sept. (1995) 1-10 